\section{Diabetes in Cystic Fibrosis}

Seif Yahia, Pramya Mahendra, Kok Kee Liew, Tun Win Hla, Adrian Paszkiewicz:

Andrew Clayton, John Kalk, Jane Dewar

\section{BACKGROUND}

Patients with Cystic fibrosis are now living longer due to advances in nutritional and medical care. As a consequence a larger number are developing related co- morbidities such as cystic fibrosis related diabetes (CFRD). CFRD is the most common comorbidity in $\mathrm{CF}$, with the prevalence being up to $50 \%$ by the age of 30 . (1)

\section{AIMS}

To assess current practice in relation to the UK CF Trust guidelines 2004 Screening for CFRD should begin at age 12 using OGTT. Patients should be treated with insulin and reviewed quarterly by MDT specialising in CFRD. HbA1C should be measured quarterly to guide further therapy. Annual screening for complications should occur in all patients older than 12 years.

\section{METHODS}

Retrospective case notes and hospital database review of adults with CFRD at Nottingham University Hospital between the years 1998 and 2014. Also data was collected about changes to weight and lung functions following 1-2 years of insulin therapy.

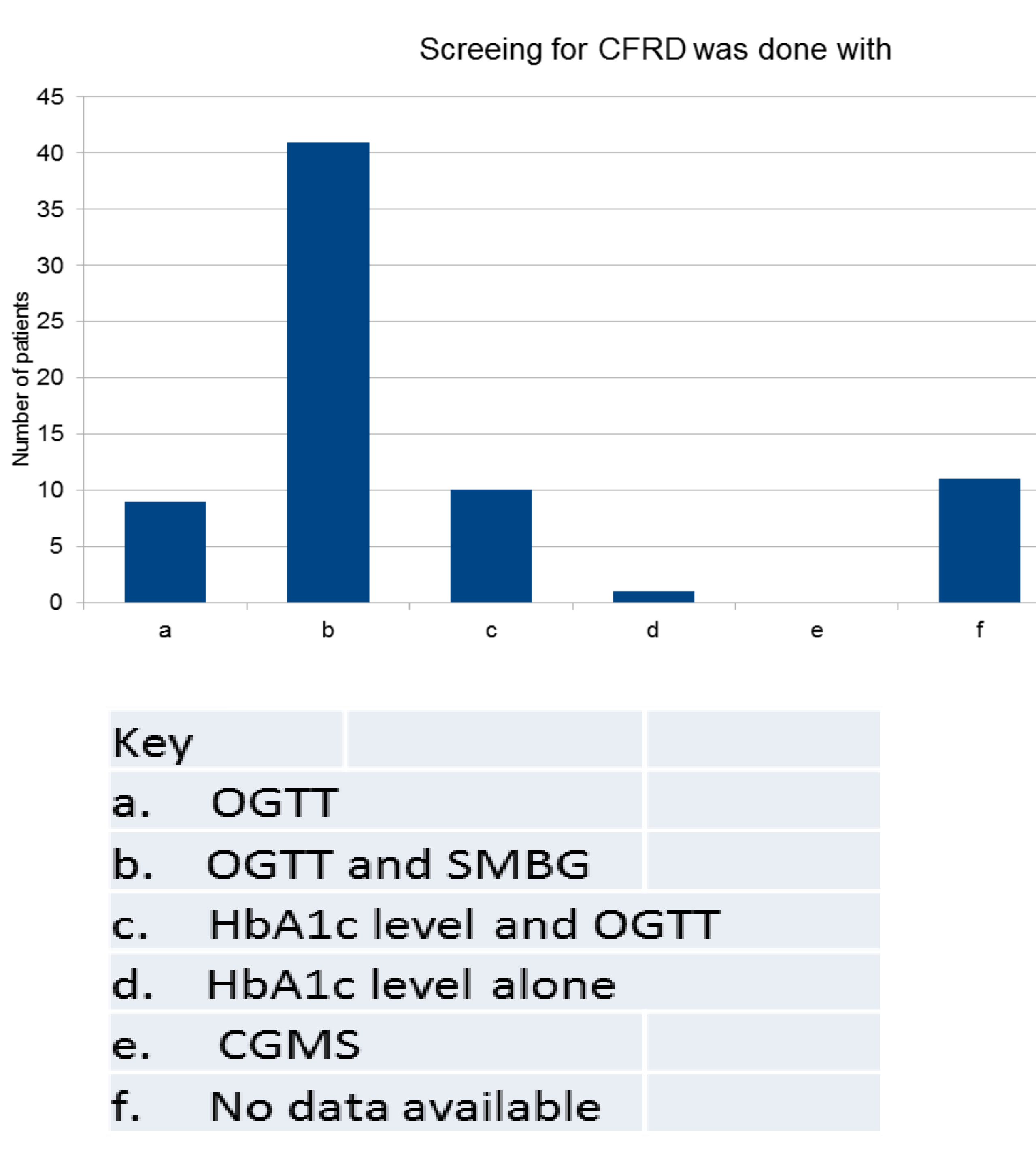

Treatment for CFRD given as:

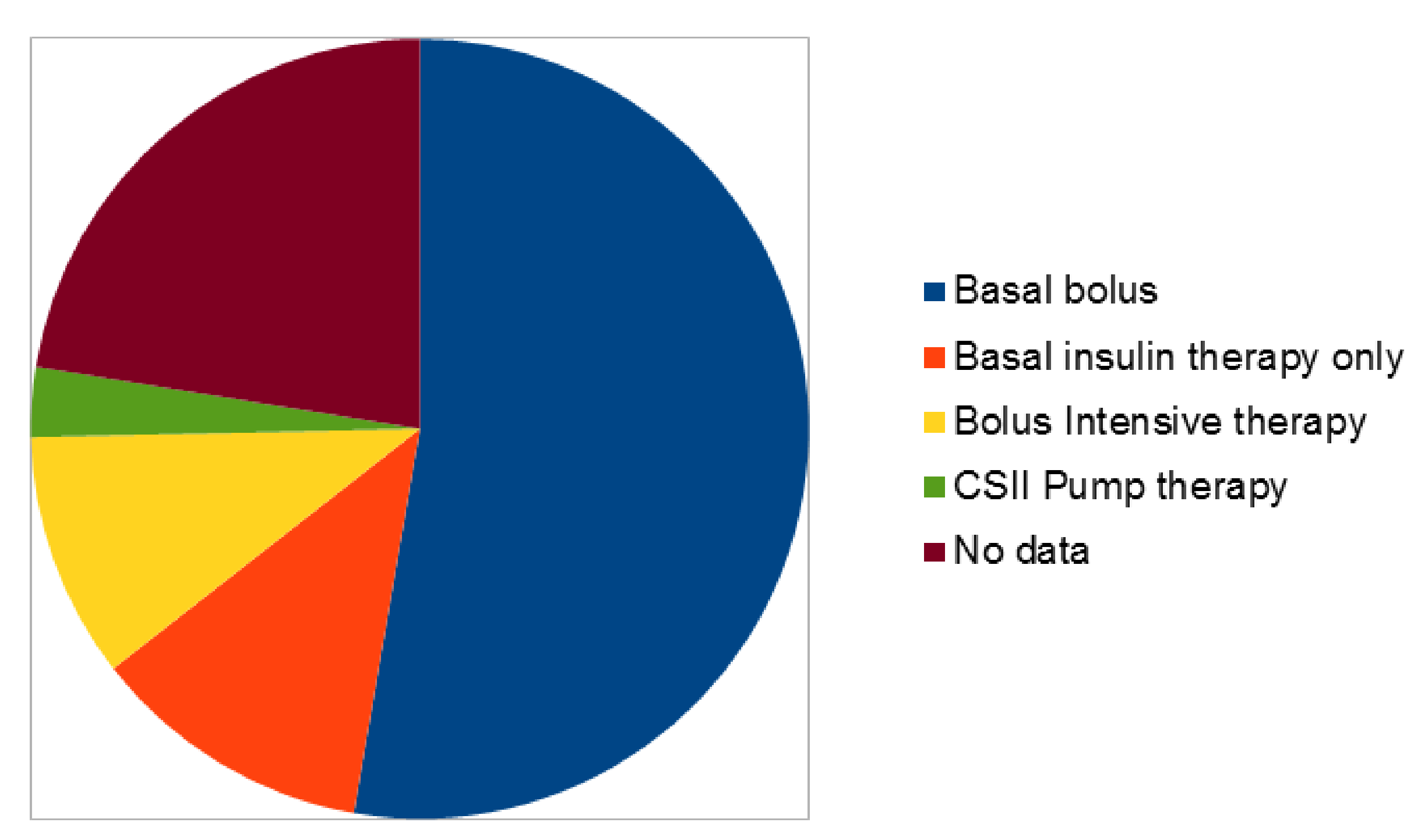

Weight/BMI after 1-2 years on insulin

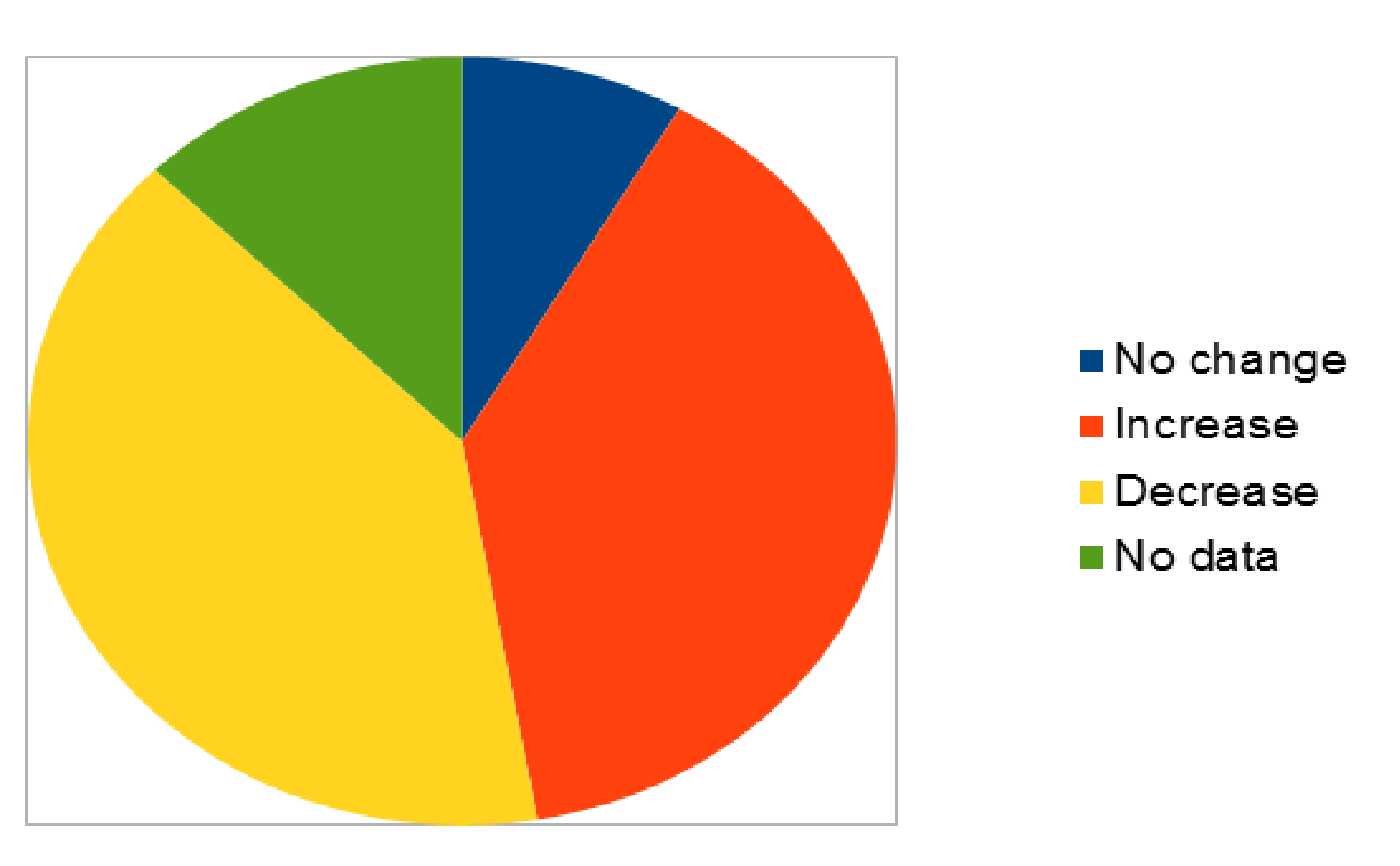

Lung Function after 1-2 years on insulin

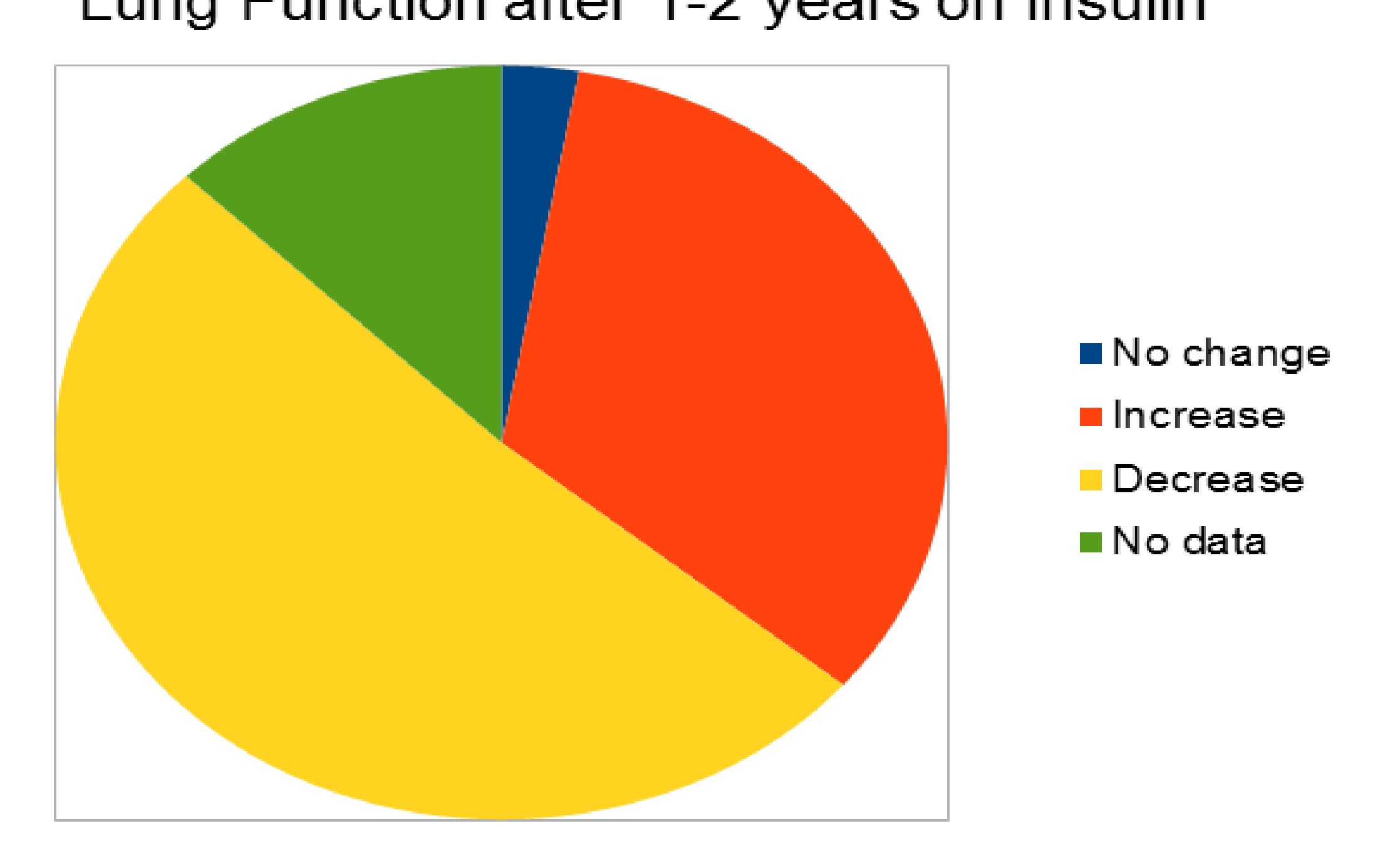

\section{DISCUSSION} 32 females). Mean age: 30 (range 1862). In $83.3 \%$ of patients CFRD was diagnosed with OGTT and patients were treated with various insulin regimens. $61 \%$ of patients were seen quarterly. $\mathrm{HbA1C}$ was measured quarterly in $41.7 \%$. Blood pressure, retinopathy, Foot, ACR, CVS events and lipid profile were screened annually in $47.2 \%, 40 \%, 25 \%, 4.2 \%$ and $45.8 \%$ respectively. Weight after 1-2 years on insulin was static in $9.5 \%$, increased in $44.4 \%$, decreased in $46 \%$, whereas, lung function was stable in $3.2 \%$, increased in $38 \%$, decreased in $58.7 \%$.

\section{CONCLUSION}

Overall, most of our patients were diagnosed with OGTT and treated with insulin. However, our performance is average in terms of follow up, monitoring and screening for complications. These discrepancies could partially be explained by the fact that data was collected from 1998 , but the guidelines were only issued in 2004. Also paediatric databases and notes were not used. Another issue with monitoring is the large number of DNAs. Nonetheless, there is likely room for improvement in these areas.
72 patients were identified (40 males,

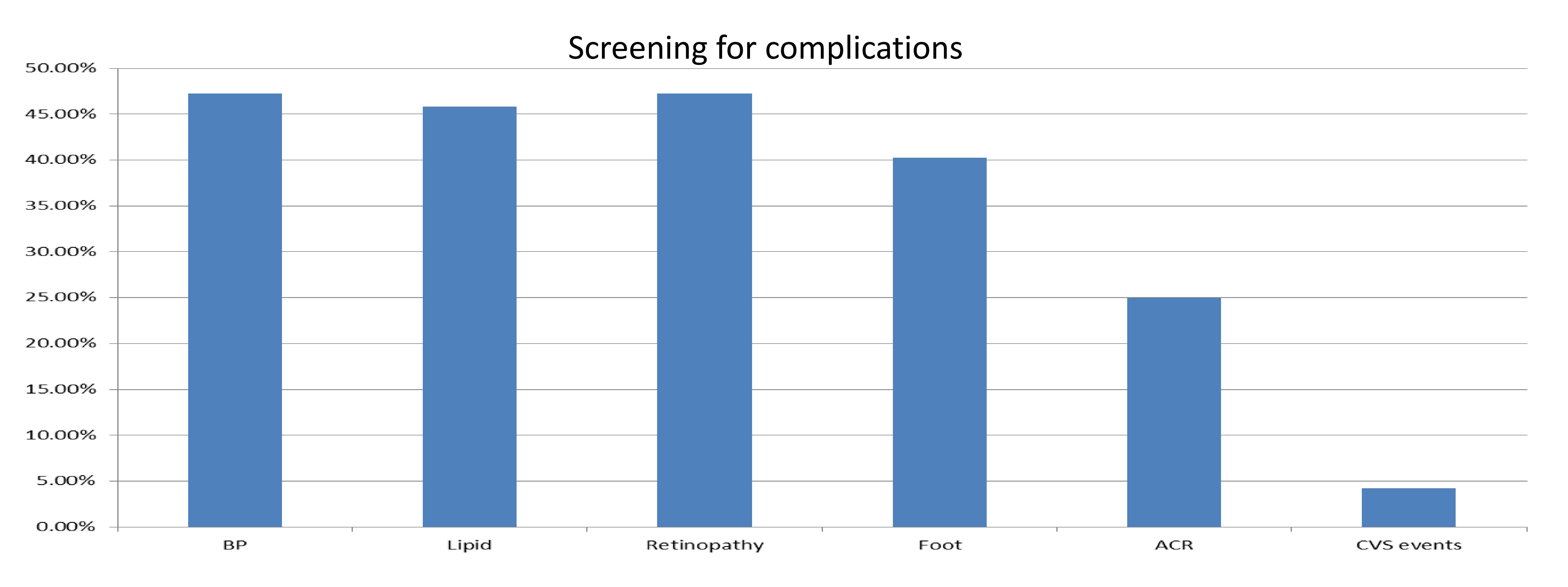

\section{REFERENCES}

(1) Moran A, Pillay K, Becker DJ et al. Management of cystic fibrosis-related diabetes in children and adolescents. Paediatric diabetes 2014; 20: 65-76. (2) Siwamogsatham O, Alvarez J, Tangpricha V. Diagnosis and treatment of endocrine comorbidities in patients with cystic fibrosis. Current Opinion 2014; 21:5. 